\title{
FACETTEN VON LATENTER VIRTUOSITÄT IM GITARRENSPIEL DES ZEITGENÖSSISCHEN FLAMENCO
}

\author{
Edin Mujkanović
}

Die Virtuosität einer hochklassigen Flamenco-Performance zieht in den Bann und zeigt sich auf der Bühne am ehesten durch rasante Staccatoläufe auf der Gitarre, die synchron mit der exzessiven Fußarbeit der Tänzerinnen und Tänzer zelebriert werden, durch komplexe und ausgefeilte Choreografien, spektakuläre kollektive Breaks sowie eine maximale Expressivität aller Beteiligten. Die internen Funktions- und Kommunikationsmechanismen, die Gewichtung von fest einstudierten und improvisierten Anteilen, die Codes und unausgesprochenen Gesetzmäßigkeiten bleiben den meisten in der Regel aber verschlossen, sofern sie nicht selber als Aficionados intensive Hörerfahrungen, oder als Gitarrist/inn/en oder Tanzende/r praktische Erfahrungen gesammelt haben. Ausgehend von der Prämisse, dass sich Virtuosität im Flamenco nicht allein anhand technischer Höchstleistungen als Ausdruck »psychomotorischer Optimierungsprozesse ( (Kopiez 2004: 212ff.) manifestiert, möchte ich mich der Frage widmen, wie sich Virtuosität im zeitgenössischen Toque ${ }^{1}$ äußert und welche Bedeutungsebenen der Begriff auf der musikalischen Ebene einschließt. Mein Ansatz geht davon aus, dass es im Flamenco nicht nur eine Form von Virtuosität gibt. Hierbei zeige ich ausgewählte Aspekte auf, die ich begrifflich als latente Virtuosität fassen möchte. Der Fokus richtet sich dabei auf einige Sachverhalte, die während des Hörens in der Regel schwieriger zu identifizieren sind, weil sie sich dem ohren- und augenscheinlichen Virtuositätsprinzip des »Schneller - höher lauter « weitgehend entziehen. Es sind die nicht offensichtlichen oder sich erst auf den zweiten Blick erschließenden bzw. nur mittels einer gewissen Hörerfahrung erkennbaren Kunstfertigkeiten und Höchstleistungen. Ich stütze mich auf Beobachtungen der zeitgenössischen gitarristischen Flamencoszene, die ich als aktiver Musiker und Forschender, also als involvierter Beteiligter im Sinne des Musicking (Small 1998) aus vielerlei Perspektiven

1 Flamenco besteht im traditionellen Verständnis aus den Elementen Gesang (Cante), Tanz (Baile) und Gitarre (Toque). 
studiere. ${ }^{2}$ Latente Virtuosität im Flamenco kann sich beispielsweise durch besondere Fähigkeiten in der kommunikativen Interaktion bei der Gesangsund Tanzbegleitung zeigen.

\section{Virtuosität als interaktive Facette in der Gesangs- und Tanzbegleitung}

Flamencomusiker/innen sind sich in der Regel einig, dass die Essenz des Toque in der Tanz- und Gesangsbegleitung verborgen ist. Innerhalb der Flamencokultur genießt eine hinreißend ausgeführte Gesangsbegleitung ein ungleich höheres Ansehen als das rein solistische Spiel. Jede/r Flamencogitarrenschüler/in in Andalusien sammelt mit dem Begleitspiel die ersten Erfahrungen und vor allem die sichere Beherrschung des Compás ${ }^{3}$. Ein guter Begleitgitarrist beherrscht alle Cantes und Bailes meist so gut, dass er sie ebenfalls singen oder tanzen könnte. Alle Gitarrenvirtuosen verfügen über ein enzyklopädisches Wissen im Hinblick auf Gesangs- und Tanzbegleitung, können selber hochklassige $\mathrm{Palmas}^{4}$ produzieren und zumeist auch einen mehr als ordentlichen Gesang abliefern.

Eminent wichtig für die Begleitung ist der Instinkt, die nächsten Schrittfolgen des Tänzers oder die Wendungen des Sängers antizipieren zu können, um mit einer adäquaten musikalischen Phrase die Stimmung der Tänzer und Sänger zu intensivieren. Dem Tocaor wird dabei die Sensibilität und Intuition abverlangt, Phrasen und harmonische Umschlagpunkte vorauszuahnen, mit angepasster Dynamik und Intensität zu agieren, und ein Gespür zu entwickeln, wann das Tempo zu beschleunigen oder wann laid back zu spielen ist. Der Gitarrist hat vielleicht die anspruchsvollste Aufgabe in einer Fla-

2 Christopher Smalls Begriff des Musicking erweist sich als hilfreich, die eigene Position als Forschender zu erklären und sich einem Komplex wie dem Flamenco zu nähern. Small revitalisierte die Idee, Musik eher als ein Verb zu betrachten und weniger als ein Nomen. Dahinter steht der Gedanke, alle sozialen Aktivitäten, die musikalischen Text und Erfahrungen produzieren, in die Analyse einzubeziehen. Musicking umfasst Komposition, Performance, Hören, Tanzen u.v.m. Dieser multiple Ansatz entspricht meiner ganzheitlichen Annäherungsweise, bei der ich Konzerte und Juergas besuche, wann immer es geht, Aufnahmen studiere, mich mit anderen Aficionados austausche, Unterrichtsstunden nehme und gebe, Workshops besuche, Magazine lese, Tanz- und Gesangsbegleitung als Gitarrist absolviere und Konzerte spiele.

3 Der Begriff des Compás ist komplexer Natur und weist zunächst auf die dem Flamenco zugrunde liegenden Rhythmusmodelle hin. Näheres folgt im Laufe der weiteren Ausführungen.

4 Die für den Flamenco typischen rhythmischen Klatschtechniken werden als Palmas bezeichnet. 
mencoformation. Er muss sein Instrument so sicher beherrschen, dass er mit seiner Aufmerksamkeit ganz bei den anderen Protagonisten sein kann.

»Dabei ist es aber nicht etwa so wie im klassischen Tanz oder im Jazz, dass der Tänzer der Musik folgt bzw. der Solist sich im Rahmen der Rhythm-section bewegt. Nein, der Tänzer bestimmt Tempowechsel, Remates ${ }^{5}$ [...] und Abfolge oft aus dem Moment heraus - genauso wie der Sänger, [der] sobald er zu singen beginnt, für die Gitarre den Ton angibt. Wir [die Musiker] haben gleichzeitig zu folgen und zu steuern; auftretende Fehler liegen grundsätzlich beim Gitarristen, da es seine Aufgabe ist, wie ein Torwart alles aufzufangen« (Iven 1990: 140).

Latente Virtuosität zeigt sich in der Fähigkeit, die anderen musikalischen Akteure glänzen zu lassen und aus dem Augenblick heraus intuitiv und risikoreich die passenden Entscheidungen zu treffen ohne sich auf die eingetreten Pfade der zweifellos vielfach vorhandenen Klischees zu beschränken. Virtuose Gesang- und Tanzbegleitung kommt ganz ohne technische Manierismen aus. Es zählen expressive und kommunikative Fähigkeiten auf der Grundlage des Erfahrungsschatzes und gleichzeitig der Mut, neue Wege zu begehen ohne die Cantaora oder den Cantaor mit dem eigenen Spiel so zu verunsichern, dass die Inspiration zum Singen verschwindet oder Einsätze nicht gefunden werden. Bei dieser Form der Virtuosität geht es auch um Höchstleistungen, aber diese basieren auf einer gruppendynamischen und kommunikativ-spielerischen Durchdringung und Bewältigung von riskanten Situationen oder zumindest wenig kalkulierbaren Erfahrungsräumen in performativen Zusammenhängen. Die Ausführung besitzt bei dieser spielerischen Erforschung von kontrollierter Freiheit Vorrang gegenüber dem Inhalt, d.h. entscheidend ist die Interpretation und Art und Weise, wie und weniger was gespielt wird.

Eine Facette der Virtuosität besteht im Wagnis, aus der ausschließlich dienenden Rolle des Begleiters herauszutreten und die eine oder andere bislang ungehörte und spektakuläre musikalische Aktion aus dem Moment heraus zu demonstrieren. Dies kann sich in kleinen Details zeigen: ein, zwei untypische Akkorde in einem ungewöhnlichen Voicing, eine rhythmische Variation, ein unerwarteter Akzent, eine ideenreiche ornamentale Figuration als Antwort auf eine Gesangsphrase oder in einem gewissen Moment auch einmal nichts zu spielen, den Mut zu atmosphärischen Lücken aufzubringen und so neue Klangräume zu eröffnen und zu erschaffen. Virtuosität heißt in diesem Sinne, im richtigen Moment das Richtige zu fühlen und in

5 Hierunter wird der so charakteristische Flamencoabschluss verstanden. Die aufgebaute gebündelte Energie und Spannung einer Passage kulminiert und entlädt sich in einem Remate. 
musikalische Handlungen und Aktion umzusetzen. Pedro Sierra ( ${ }^{* 1966)}$ aus Barcelona, Manuel Parilla (*1967) und Diego del Morao (*1979) aus Jerez sind als herausragende Vertreter einer solch virtuosen Gesangs- und Tanzbegleitung zu nennen.

\section{Virtuosität als Beherrschung der Technik}

Flamencogitarristen beherrschen eine Reihe von spezifischen Techniken, die im Repertoire der klassischen Gitarre gar nicht oder nur am Rande Verwendung finden. Spieltechnik sowie deren Ausführung im Hinblick auf einen erwünschten Ausdruck sind nicht voneinander zu trennen, »da eine Spieltechnik letztlich nichts anderes ist als die materiale Ausführungsseite einer ideellen Konzeption. Umgekehrt jedoch hängt die musikalische Ausdrucksfähigkeit ganz wesentlich von der Perfektionierung der technischen Möglichkeit ab« (Schulze 1985: 152). Die Flamencospieler messen ihrer Technik und Spielkultur einen hohen Stellenwert bei, betonen aber stets, dass die Technik nicht um ihrer selbst willen eingesetzt wird, sondern nur als Transportvehikel einer persönlichen Ausdrucksweise und damit spezifischen Klangund Spielästhetik zu betrachten ist. Obwohl das technische Niveau der Spitzenmusiker äußerst hoch ist, agieren die Musiker in dem Selbstverständnis, keine Virtuosen zu sein. Kaum jemand käme auf die Idee, sich selber mit diesem Prädikat zu versehen.

Um die zentralen Techniken stichwortartig zu benennen:

Rasgueado: Durch Auf- und Abschläge der Finger realisierte volltönende, rasante und zugleich dichte Akkordfolgen; sie sind »heart and soul of the ssound of flamenco « (Faucher 1994: 19)

Golpe: $\quad$ Schläge mit einzelnen Fingern auf die Gitarrendecke

Tremolo: Tonwiederholungen in Quintolen

Pulgar: $\quad$ angelegtes Daumenspiel

Alzapúa: „das Plektrum anheben«: schnelles Auf- und Abschlagen des Daumens

Arpeggio: Akkordzerlegungen

Tapado: $\quad$ abgedämpfte perkussive Gitarrenrhythmen der rechten Hand Picado: $\quad$ angelegter Wechselschlag; schnelle Staccatoläufe

Der Übeaufwand für die meisterhafte Beherrschung der skizzierten Gitarrentechniken ist immens. Im Fall einer gekonnten Ausführung wird das klangliche und visuelle Resultat einer Technik während einer Performance meist per se als spektakulär und virtuos wahrgenommen. Anders gesagt: Die skiz- 
zierten Techniken sind wie dafür gemacht, augen- und ohrenfällige Virtuosität zu demonstrieren. Schnelligkeit in Form von sauber und rasant ausgeführten Picados oder Alzapúas als Element zur Intensivierung des Ausdrucks genießt eine gewisse Anerkennung unter den Tocaores. Es stellt eine große Herausforderung dar, die Techniken schnell und sauber auf einem konstanten Level flüssig in verschiedenen Spielsituationen zu absolvieren. Die Beherrschung dieser Elemente entspricht dem Kern der allgemeinen Vorstellung vom Virtuosen, der die schnellsten und waghalsigsten Läufe in scheinbarer Leichtigkeit spielen kann.

Die aktuelle Generation der Gitarristen beherrscht die virtuosen Techniken bereits mit jungen Jahren und betrachtet dies als selbstverständliche Voraussetzung. Mit zunehmender musikalischer Reife und fortschreitendem Lebensalter liegt der Fokus dann eher auf Aspekten der Komposition, der Musikalität sowie auf der Schöpfung einer individuellen Ausdrucksweise, eines eigenen Ideolekts. Die hier umrissene technische Virtuosität bildet im Kern nur die Grundlage für die tatsächliche Meisterschaft, die sich als eine der im Folgenden skizzierten Facetten von Virtuosität präsentieren kann.

\section{Virtuosität als vollkommene Internalisierung des Compás}

Im Flamenco ist der Compás das Maß der Dinge. Der Begriff besitzt zum einen die Bedeutung von Rhythmus im Allgemeinen, zum anderen bezeichnet er den charakteristischen Rhythmus eines bestimmten Cante inklusive seiner Akzente (z.B. Compás de Bulerías). Der Compás ist die allen Flamencoformen immanente Ordnungsstruktur, welche ohne Unterbrechung von Anfang bis Ende wiederkehrt und einem Ostinato vergleichbar wäre (Manuel 2006: 102). Gleichwohl gestaltet sich das Konzept Compás viel komplexer und impliziert daneben harmonische Wechsel, rhythmische Spannungsmuster, timbrale Aspekte und melodische Formeln, die den Grundgestus eines Palo

6 Unter Palos sind die verschiedenen stilistischen Gattungen des Flamenco zu verstehen. Sie bilden die musikalische Grundlage und unterscheiden sich hinsichtlich verschiedener Parameter. Hierzu zählen u.a. Metrum, Rhythmus, Melodie, Harmonieschemata, tonaler Bezugspunkt, Tempo, Textinhalt und Aufführungspraxis. Alle Palos kennzeichnet zudem eine eigene qualitative Atmosphäre, ein bestimmtes definiertes Flair und ein Charakter, welcher im Flamenco mit dem Begriff Aire beschrieben wird. Dazu existieren unzählige personelle und regionale Ausprägungen der einzelnen Gattungen. Der Name einer Form definiert traditionell die soeben aufgezählten Parameter. Jeder Palo gibt ein Grundgerüst vor, innerhalb dessen die ausführenden Künstler ihre individuellen Versionen realisieren können. 
im Flamenco definieren: »Compás communicates emotion and mood« (Landborn 2015: 86). Es ist der gemeinsame Herzschlag oder der Teppich, auf dem die Musiker/innen zum Flug animiert werden.

Der zyklische Charakter des Compás wird gerne anhand einer Flamencouhr veranschaulicht. Hierbei werden die zwölf Schläge als Ziffern einer Uhr dargestellt. Der Zeiger dreht sich kontinuierlich im Kreis und passiert immer alle Ziffern.

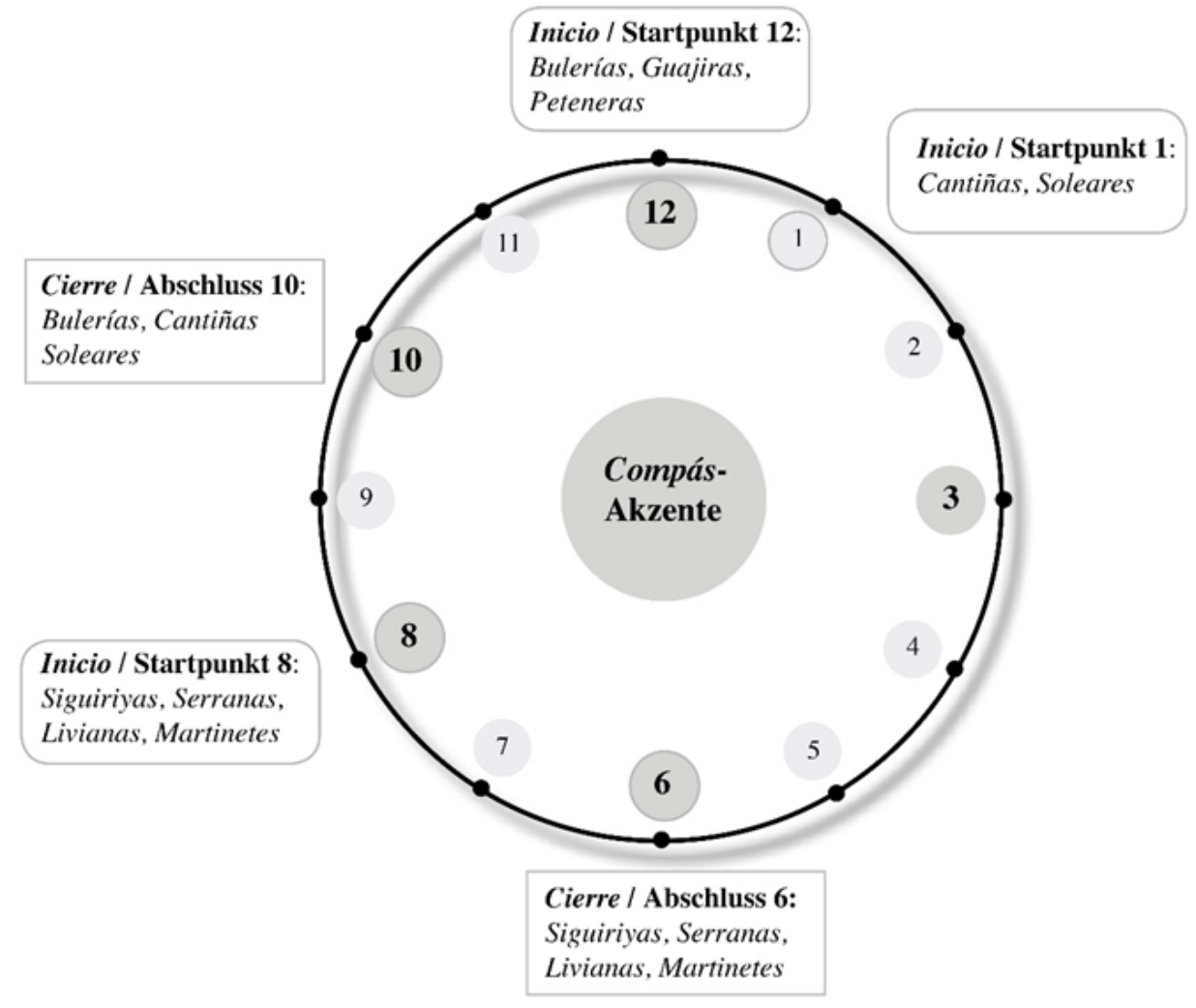

Abbildung 1: Compás-Uhr

Die Flamencouhr ist ein simplifizierendes Modell, denn es bedeutet nicht, dass die tatsächlich gespielte Musik fortwährend genau den Akzenten des Schemas folgt oder die Bulerías immer im 12er-Puls gedacht, gefühlt, gespielt, gelernt oder gelehrt wird. Der flexible 12er-Compás der Bulerías beispielsweise lässt unendliche Möglichkeiten zu, sich künstlerisch auszuleben. Der musikalische Bogen dieses Pulses birgt eine inhärente Tendenz zur rhythmischen Verdichtung und lässt gleichzeitig viel Spielraum für Binnendifferenzierungen, Akzentverschiebungen, Flexibilität und improvisatorischen Freiraum. Viele Musiker zählen gar nicht, sondern spüren einfach oder nehmen allenfalls auf den 6er bzw. 3er Puls (ritmo de tierra) Bezug. Die Uhr suggeriert außerdem, dass die musikalischen Phrasen immer oder in der Regel auf der Zählzeit 12 beginnen und auf der 10 stoppen. Die Vielfalt der heutigen Interpretationen einer Bulerías zeigen jedoch, dass die Musiker 
sich auch von diesen traditionellen Betonungsschemata stark emanzipiert haben. Der Einstieg (Inicio) der Falsetas ${ }^{7}$ oder in die Paseos als charakteristische Akkordfolge, die den Palo definieren, wird im Prinzip auf jeder Zählzeit realisiert. Die gleichsam kraft- wie wirkungsvollen Abschlüsse (Remates) werden entweder direkt klassisch auf die Zählzeit 10 oder synkopisch davor gesetzt, oder eben auch auf die 9 oder 8+ oder 8, manches Mal auf die 4+ oder die 11+. Die Akzenten und Abschlüsse werden spielerisch platziert: Je virtuoser die ausführenden Musiker/innen, desto überraschender, abenteuerlicher und scheinbar völlig ins Off gesetzt sind die Akzente letztlich. Bulerías ist letztlich ein permanentes rhythmisches Spiel, bei dem sich je nach beteiligten Akteuren verschiedene rhythmische Schichten und damit auch Akzente überlagern können.

Der Stellenwert einer totalen Compás-Sicherheit kann gar nicht hoch genug eingeschätzt werden. Die Metapher eines permanent rotierenden Rades hilft zu verstehen, dass ein grundlegender Aspekt der Virtuosität im Flamenco darin besteht, in jeder Situation auf das drehende Compás-Rad auf- und abspringen zu können und zu jeder Zeit intuitiv zu wissen, an welchem Punkt oder in welcher Phase der Umdrehung man gerade ist. Virtuosität heißt, dass der Compás in allen maßgeblichen Palos völlig internalisiert ist und die Musiker/innen absolut frei von metrisch-rhythmischen Restriktionen oder Hemmnissen agieren. Jede und jeder, der sich in Tanz oder Gitarrenspiel bereits mit den Grundlagen oder Feinheiten des Compás auseinandergesetzt hat, weiß um die Herausforderung dieses für viele zumeist lebenslangen Lernens, nämlich einerseits a compás zu sein und andererseits innerhalb dieses strikten Regiments Freiheit zu erlangen und sich nach Belieben bewegen und artikulieren zu können.

\section{Virtuosität als meisterhafte Beherrschung des Contratiempo}

Eines der entscheidenden Merkmale des modernen Toque liegt in der rhythmischen Ausformung. Das Prinzip des Compás als metrische Grundformel der Zeitgestaltung ist weitgehend unangetastet geblieben, aber die Spielpraxis basiert deutlich auf einer starken Offbeat-Phrasierung durch synkopischvertrackte Akzentverschiebung. Dieses Spiel »gegen den Beat« oder »gegen

7 Falsetas sind die üblicherweise von den Gitarristen auskomponierten Kompositionsbausteine. Ihre Länge ist variabel und es kann sich dabei um eine solistische melodische Phrase oder der Kombination aus Melodie- und Akkordspiel handeln, die z.B. nach einer Gesangseinlage eingeleitet wird. 
die Zeit « bezeichnen die Flamencos als Contratiempo. Die Einführung gröBerer Ensembles mit Begleitgitarren, Palmeros sowie Perkussion inklusive ein oder gar zwei Cajónisten hat die Gitarre von der früheren rhythmischen Funktion, den Compás zu markieren, weitgehend entlastet. Gleichzeitig hat dadurch die Vielfalt an von der Gitarre gespielten komplexen Phrasierungsmustern, Gegenrhythmen und polyrhythmischen Prozessen enorm zugenommen und kann inzwischen als das Markenzeichen des modernen Gitarrenspiels gelten. Die Falsetas besitzen nicht mehr den durchlaufenden Charakter vergangener Zeiten, sondern zeichnen sich ebenso durch eine breite Entfaltung der dynamischen Möglichkeiten aus. Das zeitgenössische Toque lässt Lücken und bietet dynamische Kontraste durch zwischenzeitliche Stille und unvermittelt eintretende explosionsartige Steigerungen der Lautstärke und Intensität an bestimmten Punkten. Einer der musikalisch einflussreichsten Gitarristen, der diese Spielweise perfektioniert hat, ist der in Sevilla geborene, aber in Córdoba verwurzelte Gitarrist Vicente Amigo (*1968). Seine Melodien zeichnen sich durch eine dezidierte Offbeat-Phrasierung (Contratiempo) aus. Ganz typisch sind Bindungen (Ligados) zur schweren Zählzeit hin sowie synkopische Antizipationen von Zieltönen, die dann von nachschlagenden Akkorden gestützt werden. Der Ausschnitt der Falseta aus »Campo de la Verdad« (s. Abb. 2) demonstriert das Arpeggiospiel als eine der herausragenden Techniken dieses Musikers. Hierbei werden immer wieder einzelne Töne rhythmisch vorgezogen, auf die nächste Zählzeit übergebunden und durch das angelegte Spiel des einzelnen Ringfingers herausgehoben. Über den Compás bildet sich so durch die Akzente in den Arpeggios eine zweite unabhängige Rhythmusschicht, ein fast polyrhythmischer Effekt zum Beat der Cajón bzw. Palmas. Im Klangbild hat dies einen luftigen und fließenden Charakter zur Folge. Im weiteren Verlauf werden von Compás zu Compás-Durchlauf nicht nur die Harmonien, sondern auch die Zerlegungsmuster verändert.

Das Contratiempospiel zeigt sich noch ausgeprägter in dem für Vicente Amigo so charakteristischen langsamen Bulerías-Typus mit dem Titel »Río de la Seda« vom Album Tierra. Die Transkription (s. Abb. 3) zeigt, dass die Einsätze der Melodie und die Akkordakzente zum Ende eines jeden Compás permanent synkopisch gesetzt sind. Die Art der Phrasierung in Bezug auf den Puls ist so galant-leichtfüßig wie beständig ins Offbeat gesetzt, dass der Eindruck entsteht, das Stück sei durchgängig um eine Achtel versetzt zum Puls angelegt. In dieser Anlage von zwei übereinander gelegten rhythmischen Schichten, in der in einem unaufgeregten Duktus elegante und mühelos schwebende Falseta-Linien viel Luft und Raum lassen, zeigt sich Virtuosität par excellence. 

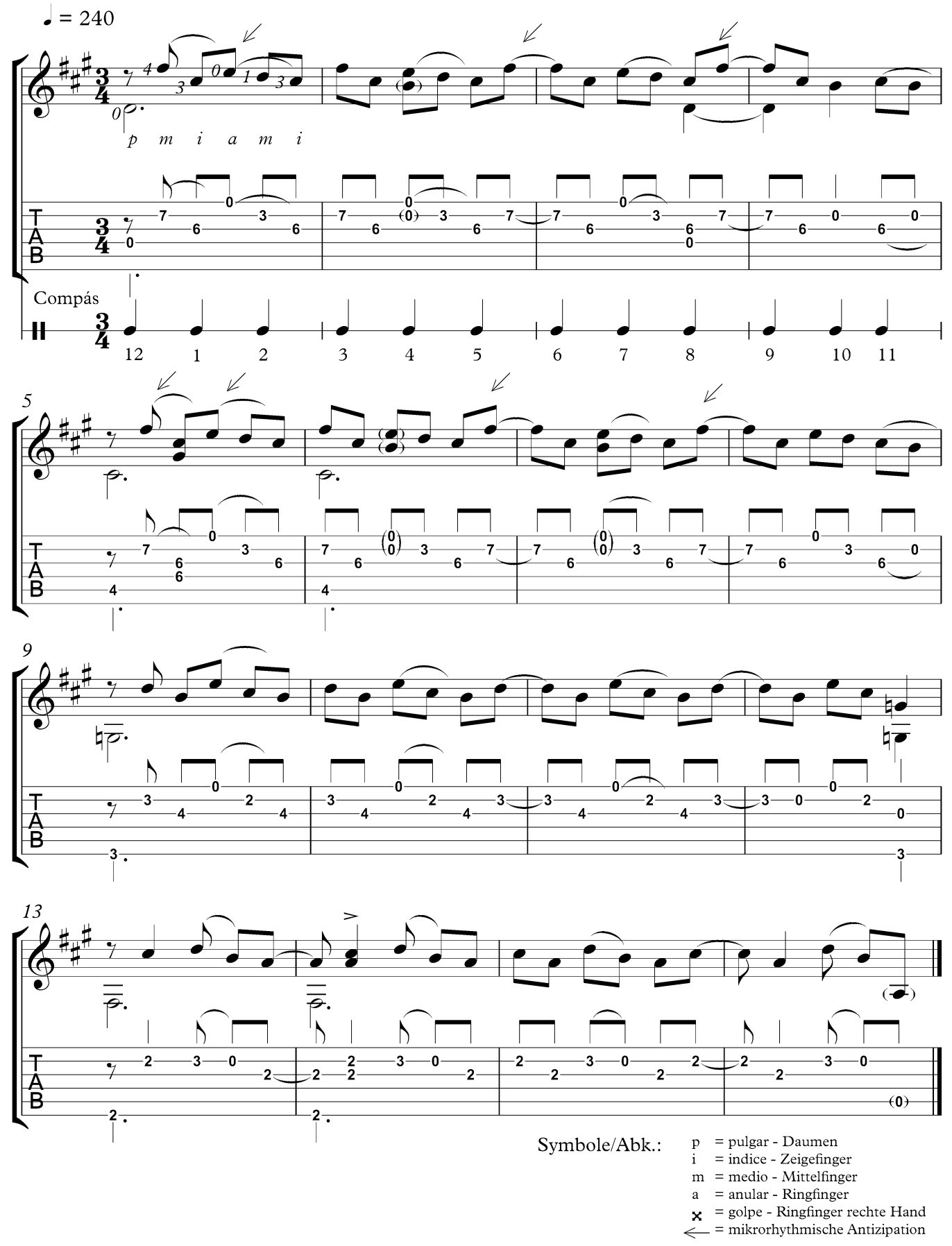

Abbildung 2: Vicente Amigo - »Campo de la Verdad«, Falseta 2, ab 2:398

8 Die Notationen und Transkriptionsmodi von Flamencomusik sind immer wieder Gegenstand von Diskussionen. Im Falle der Bulerías habe ich mich der besseren Lesbarkeit wegen bewusst für die Schreibweise von vier Dreivierteltakten für einen Compás-Durchgang entschieden. Die übliche Anfangszählzeit 12 wird hier als Taktanfang und Schwerpunkt notiert. Um die Flamenco basierte Logik von Bewegungen und Voicings auf dem Griffbrett besser nachvollziehen zu können, ist zusätzlich das Tabulatursystem angegeben. 
$\cdot=230$

Kapo 1. Bund
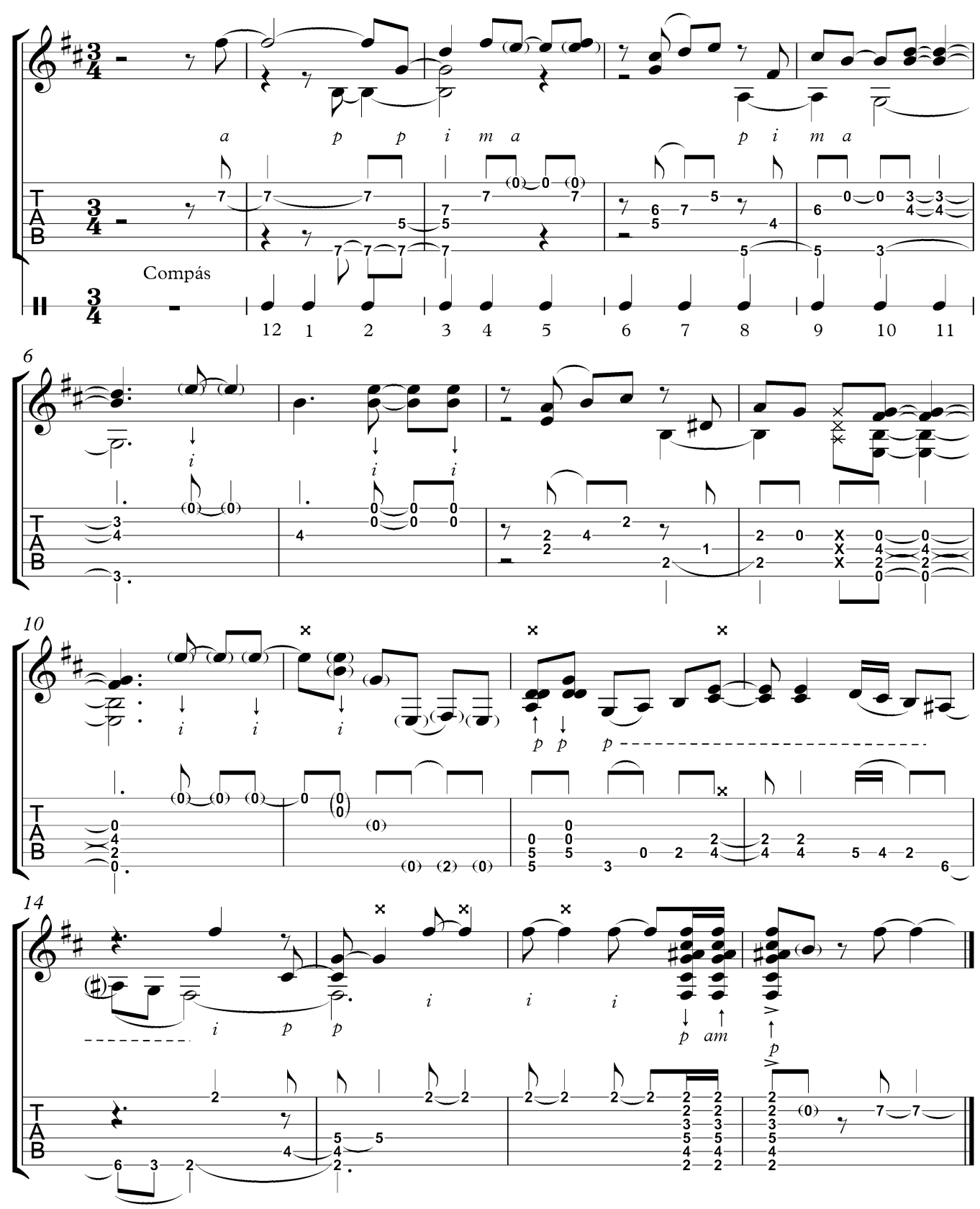

Abbildung 3: Vicente Amigo - »Río de la Seda«, Falseta 1, ab 1:489

Das Beherrschen des Contratiempo ist das Schlüsselelement des zeitgenössischen Flamenco und dies gilt im Besonderen für die Bulerías. Die Kultivierung des Contratiempo ist für die Künstler das Mittel, ihre Meisterschaft in Form einer völligen Kontrolle dieser Komponente zu beweisen. Der Compás und die auf seiner Basis entstehenden Melodien werden durch Contratiempo zum Leben erweckt. Dieses skizzierte gehobene Spiel ist gewiss dem Einfluss

9 Das Beispiel wird mit dem Kapodaster im ersten Bund gespielt und klingt damit einen Halbton höher als notiert. 
und Erbe Paco de Lucías (1947-2014) zuzuschreiben. Er demonstriert Mitte der 1970er Jahre als einer der ersten eine gänzlich neue Phrasierung und rhythmische Auffassung im Flamenco, in der auf der Grundlage des Compás Melodien auf einer neuen unabhängigen musikalischen Ebene entwickelt werden. Er brach das starre Korsett des Compás auf und emanzipierte die Melodie als eigenständige Komponente unabhängig von der Gravitation des Compás. Künstlerisch und spielerisch bedeutet dies einen enormen Zuwachs an melodischer Freiheit. Wichtig festzuhalten ist, dass sich all die scheinbar schwerelos mäandernden Melodien nur gegen einen gleichbleibenden Puls als Referenz entwickeln können. Dieser solide Puls verlangt selber nach einem speziellen Feeling in der Ausführung, bei dem der Musiker seinen persönlichen Swing und Groove (Aire/Soniquete) auch als individuelles Alleinstellungsmerkmal etablieren kann.

Eng verbunden mit dieser rhythmischen Komponente ist der virtuose Umgang mit dem oben erwähnten Element der Dynamik. Vor allem im LiveEinsatz wird das rhythmisch-dynamische Spiel perfektioniert und spiegelt in fast ritualisierter Form das Erscheinungsbild des modernen Flamenco wieder. Paco de Lucías Art, rhythmische Tapados bis an die maximale Spannungsgrenze zu spielen, um danach mit einem spektakulären Rasgueado oder Picado die melodischen Themen zu eröffnen, setzte Maßstäbe im Toque und hat Gitarristen wie Vicente Amigo und viele andere nachhaltig geprägt. Die hochgetriebene Spannung der rhythmischen Muster der Gitarren und oft zweier Cajónisten entlädt sich in fulminanten Akzentfeuerwerken in den Remates, um dann augenblicklich in Momente völliger Stille zu münden. Diese kollektive Dynamisierung der Rhythmik ist ein wichtiges Merkmal der Spielpraxis des modernen Flamenco und hat entsprechend bestimmte Erwartungshaltungen beim Konzertpublikum verankert. Ich würde sogar einen Schritt weiter gehen und die These wagen, dass sich die Evolution der virtuosen zeitgenössischen Flamencogitarre vor allem in diesem skizzierten Feld abspielt.

\section{Virtuosität als Kreation von frictions}

Musikalische Entscheidungen basieren in Bezug auf einen musikalischen Stil häufig auf der Annahme von operativen Regeln und dem Umstand, dass diese Regeln gebrochen werden können. Diese Regeln können in allen musikalischen Domänen existieren: Rhythmus, Harmonien, Melodie, Produktion, Instrumentation. Allan Moore präferiert den Begriff der Normen und ersetzt das Konzept des Regelbruchs durch eine Idee, bei der es um die »creation of 
friction « (Moore 2013: 167f.) geht. Frictions werden in diesem Sinne als Spannungen oder Reibungen verstanden zwischen akzeptierten Normen eines Stils bzw. dem, was der Hörer in Abhängigkeit von seinem Grad an Kompetenz zu hören erwartet und dem, was letztlich in einem Track passiert. Jede auftretende Differenz zwischen beiden Polen kann als friction gedeutet werden, welche zugleich immer affektive Werte beinhaltet, über die sich formidabel diskutieren lässt. Gerade am Beispiel von Flamencogitarristen lassen sich frictions zwischen akzeptierten Normen und realen Erscheinungsformen bestens darlegen. Die Virtuosen des Flamenco spielen virtuos mit der Möglichkeit, konstant Spannungen zwischen kanonisiertem Vokabular und ihren individuellen kreativen Impulsen und künstlerischen Ansprüchen zu kreieren, da ihr Fokus sich nicht mehr ausschließlich auf die Überwindung von technischen Limitationen richtet.

Ein Musiker, der im traditionellen Flamenco verwurzelt ist, aber gleichzeitig keinerlei Berührungsängste mit anderen Gattungen wie Jazz, Funk, Pop und Latin kennt, ist der aus Jerez de la Frontera stammende Gerardo Núñez (*1961). Er ist einer der großen Innovatoren des Flamencospiels, der mit zahlreichen Jazz- und Fusion-Musikern zusammenarbeitete und den Flamenco-Jazz maßgeblich mitgestaltete. Ihn nur darauf zu reduzieren, wäre aber unangemessen, denn für ihn und andere Virtuosen der Flamencogitarre ist kennzeichnend, dass sie unterschiedlichste Spielkonzepte verinnerlicht haben und je nach Kontext variabel bedienen können. In den eher traditionell ausgerichteten Liebhaberclubs, den Peñas, pflegen sie unter Umständen eine erdige und schnörkellose Spielweise, aufgelockert mit knackig-punchigen Pulgar-Falsetas. In einem oft kurzfristigen angesetzten Tourkontext liegt der Fokus wegen der kaum vorhandenen gemeinsamen Proben eher im improvisierten Spiel und in Adhoc-Arrangements, während im Rahmen der eigenen CD-Produktion schließlich das volle Potential an Innovation, Grenzgängertum und Avantgarde ausgelebt werden kann.

Das Stück »Compás Interior« aus dem Jahr 2012 ist von Gerardo Núñez mit Soleá-Nana als Palo-Bezeichnung überschrieben (s. Abb. 4). Die charakteristische E-Phrygisch-Tonalität der Soleá wird hier durch die in Scordatur (C-G-D-G-H-E) gespielte Gitarre aufgegeben, da C-Dur als tonales Zentrum fungiert. Das nur etwa 1:20 lange Stücke ist ein Beispiel für einen sehr freien, unkonventionellen Umgang mit der traditionellen Form der Soleá. Die eher traditionelle Auffassung der Gattungen (Palos) im Sinne von »Gussformen, geprägt durch die Tradition « (Caballero 1995: 47) impliziert eine verbindliche Festlegung im Hinblick auf einen emotionalen Grundgestus, rhythmische Akzente, Tonalität etc. Gerardo Núñez wiederum ist ein Vertreter, der diese Zuschreibungen aufweicht und mit ihnen spielerisch und kreativ 

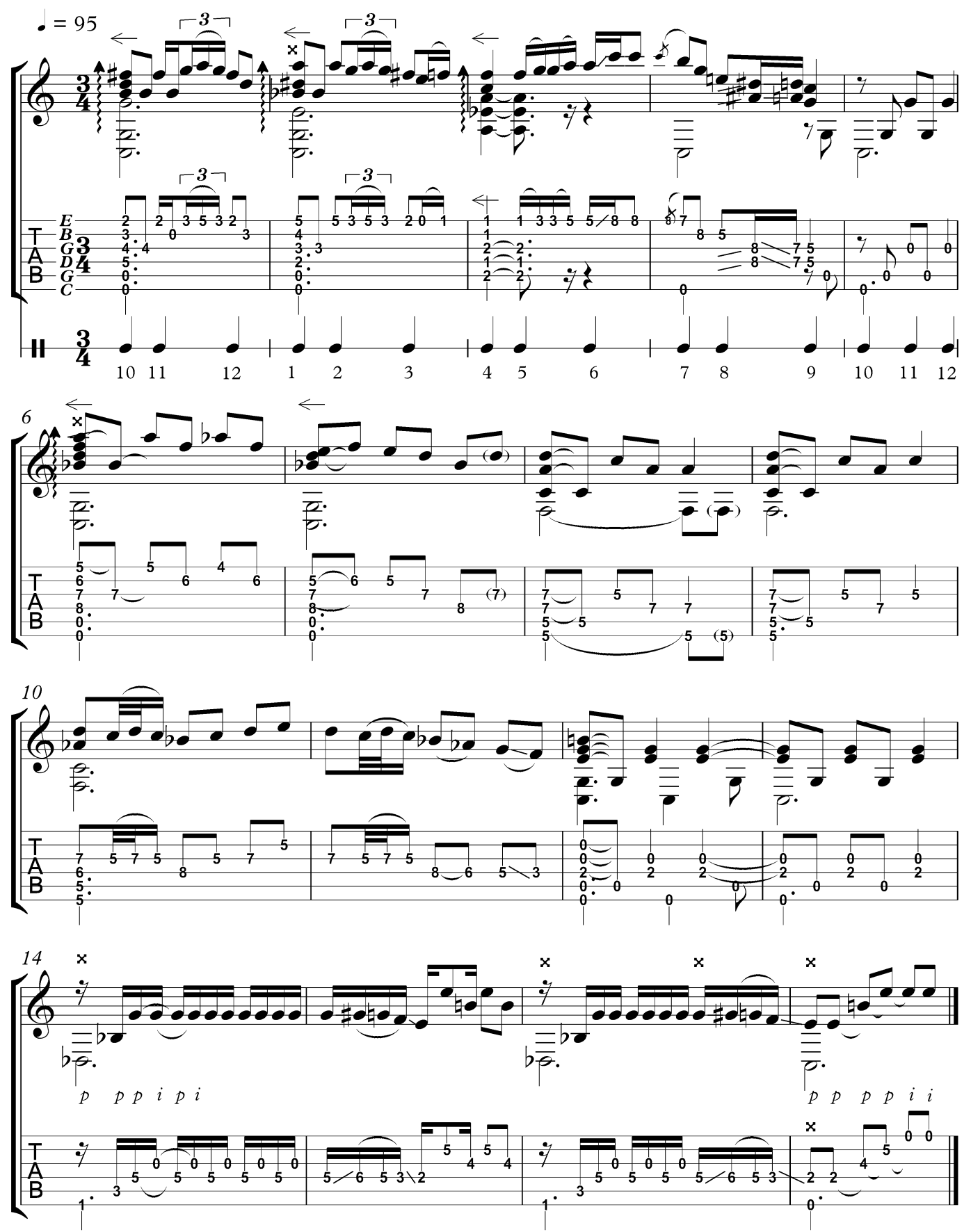

Abbildung 4: Gerardo Núñez - »Compás Interior«, ab 0:52

umgeht. Im Falle des Transkriptionsbeispiels ist die Soleá in Hinblick auf das Tempo und den selbstreflektierenden und introvertierten Charakter noch zu erahnen. Die für eine Soleá ganz und gar untypische Tonalität, der fehlende phrygische Modus, die realisierten harmonischen Bewegungen, die ausbleibende andalusische Kadenz, die asymmetrische Phrasenbildung (s. Takt 1: Phrase beginnend auf der Zählzeit 10) sowie das fehlende typische Akzentschema demonstriert dagegen einen sehr freien Umgang mit dem Formenvokabular. Subtile Bezüge zur Soleá existieren zwar, denn er spielt verein- 
zelt für diesen Stil typische Figuren einschließlich der Remates (s. Takt 1417 ), belässt es aber bei diesen Andeutungen und stellt es der Hörerin und dem Hörer frei, zu verstehen und zu entscheiden, was dieses Stück nun zur Soleá macht oder nicht. Das Stück ist technisch mit einiger Spielerfahrung auf der Gitarre umzusetzen und die Frage, was an dem Stück nun handwerklich virtuos sein soll, ist sicherlich berechtigt, weil es nicht der allgemeinen Vorstellung von Virtuosität entspricht. Die Virtuosität besteht nicht zuletzt im Mut zum Spiel mit den Grenzen. Dieses Spiel wird nachträglich dadurch legitimiert, da die nächste Generation von Tocaores es bereitwillig nachahmt, variiert und spielerisch weiterentwickelt.

Das vorliegende Stück weist auf zwei relevante Aspekte des zeitgenössischen Toque im Kontext von Virtuosität hin: Anfang der 1990er Jahre vollzogen einige Gitarristen einen bewussten Bruch mit den überlieferten Normen der tonalen Zuordnungen. Gerardo Núñez gebrauchte in seiner Siguiriya »Remache«, üblicherweise por medio (A-Phrygisch) ausgeführt, den Modus por Granaína (H-Phrygisch). Das düstere und verzweifelte Aire des Palos wird zwar durch die >Quasi-Monotonie der auf $H$ herunter gestimmten E-Saite unterstrichen, für die Aficionados war die Aufnahme dennoch ein ästhetischer Schock, da sie den Palo aufgrund der für sie klanglich ungewohnten Tonartencharakteristik nicht mehr auf Anhieb als Siguiriya identifizieren konnten (Torres Cortés 2006: 22f.). Claude Worms bezeichnet den Ansatz, die Verbindung mit den traditionellen Tonarten aufzugeben, beziehungsweise Tonalitäten neu zuzuordnen als »Metaflamenco « oder »toque en segundo grado« (Claude Worms, zit.n. Torres Cortés 2006: 22). Diese Praxis, erstmalig durch Paco de Lucía im Jahr 1972 mit dem Fandangos »Canastera« in Rondeña-Stimmung ${ }^{10}$ angewandt, breitet sich unter den Tocaores verstärkt aus. Mittlerweile existieren etliche Palos por Rondeña: Bulerías por Rondeña (Manolo Sanlúcar: »Tercio de Vara«, 1998), Rumba por Rondeña (Gerardo Nuñez: »La Habana A Oscuras«, 2004) und viele mehr. Gerardo Nuñez verwendet in seinem Stück »Soleá de la Luna Coja (2004) die Rondeña-Stimmung in C\#, spielt aber in der Tonart D-Phrygisch. Dies ist eher ungewöhnlich, da die Musiker in der Regel in der durch die Gitarrenstimmung vorgegebenen Tonart bleiben.

10 Der Gitarrist Ramón Montoya (1879-1949) etablierte in den 1930er Jahren die Rondeña als einen eigenen Palo. Die Rondeña verwendet die alte Lautenstimmung $D-A-D-F \#-H-E$ (von der tiefsten zur höchsten Saite) und stellt eine reine gitarrenbasierte Soloform dar, ein Toque libre mit einem freien Dreierpuls in C\#-Phrygisch. Der Faszination dieser Stimmung kann sich kaum ein Flamencogitarrist entziehen, weil voll und dicht klingende Harmonien mit einem Minimum an Greifaufwand zu erzielen sind. 
Auch Vicente Amigo überwand zu Beginn seiner Karriere die traditionellen Hörgewohnheiten, indem er seine Granaína »Morente im Modus D\#Phrygisch spielte, ebenso wie die Soleá por Bulería »Reino de Silia«. Seine Alegrías »Maestro Sanlúcar « wird im Modus der Minera (G\#) und die Bulerías »El Mandaíto « in C\# ausgeführt. Mittlerweise sind diese Tonalitäten ganz selbstverständlich in den Kanon aufgenommen, wobei das traditionelle Verhältnis der Palo/Tonalität-Zuordnung weitgehend aufgehoben ist, da neue Kompositionen heute in allen möglichen Modi komponiert werden. Willkür, Dekonstruktion oder gar ein Verlust der Identität werden dennoch nicht Einzug halten, da dem Flamenco durchaus die Fähigkeit zur Selbstregulation innewohnt. Virtuosität zeigt sich auch am Augenmaß und Bauchgefühl der Protagonisten. Experimentierfreudigkeit und Genre- oder Gattungserweiterung stehen meist in gesunder Balance mit traditionellen Formen- und Spielvokabular. Die bisherigen Plattenveröffentlichungen von Gerardo Núñez etwa bestätigen dieses Gesamtbild anschaulich.

Ein zweiter nennenswerter Aspekt bezieht sich auf alternative Gitarrenstimmungen (Scordaturas) im Flamenco. Nach einer langen Phase der seit Ramón Montoya bewährten Tonarten por arriba (E-Phrygisch), por medio (A-Phrygisch), por Taranta (F\#-Phrygisch), por Granaína (H-Phrygisch), por Minera (G\#-Phrygisch) und der C\#-Tonalität in der Rondeña-Stimmung etablierten sich mit den Musikern Paco de Lucía, Manolo Sanlúcar (*1945) und Victor Monge, genannt »Serranito « $\left({ }^{* 1942)}\right.$ neue Tonarten wie der C\#-Modus in Normalstimmung, D\#- sowie D-Phrygisch. Dieser Prozess vollzog sich über einen längeren Zeitraum, denn die Gitarristen, die diese Modi aufnahmen, mussten dieses für sie tonale Neuland erst mit entsprechenden Fingersätzen und Variationen erkunden und internalisieren. Auch die Hörer gewöhnten sich an die neuartigen Kadenzen, um sie schließlich als Teil des Kanons zu akzeptieren. Neben dem Zuwachs an Tonalitäten in den einzelnen Palos sind vor allem neue Gitarrenstimmungen Ausdruck des zeitgenössischen virtuosen Spiels. Die Stimmungen bedeuten, neben einer Erweiterung der Register der Gitarre, die Möglichkeit zu neuen Griffkombinationen und damit neuen bislang ungehörten und unerwarteten Harmonien und Akkordfolgen mit entsprechenden charakteristischen Dissonanzen durch die Verwendung der Leersaiten. Die Vorteile der Scordaturas im Hinblick auf die klangerweiternden Möglichkeiten und Akkordschattierungen, auch vermehrt dissonanter Natur, beschränken sich in der Regel auf eine Tonart.

Im Folgenden ist eine Übersicht gebräuchlicher Stimmungen aufgeführt. Die Angabe der Töne erfolgt von der tiefsten zur höchsten Saite der Gitarre: 
C-A-D-G-B-E: $\quad$ Gerardo Núñez - »Siempre es Tarde« (Bulerías;

Dropped C-Tuning)

C-G-C-G-B-E: $\quad$ Tomatito - »Barrio Santiago (Bulerías)

Gerardo Nuñez - »Los Caños de la Meca« (Bulerías)

Gerardo Nuñez - »Compás interior « (Soleá-Nana)

$D-A-D-F \#-A-D: \quad$ Diego del Morao - »Gañanía« (Soleá)

$D-A-D-F \#-B-E: \quad$ Ramon Montoya, ca. 1930 (Rondeña-Stimmung)

$D-A-D-G-A-D: \quad$ Dani de Morón - »Immigración« (Bulerías)

$D-A-D-G-B b-D: \quad$ Tomatito - »Caminillo Viejo « (Tangos)

$D-A-D-G-B-E: \quad$ Ramon Montoya, ca. 1912-1927 (Dropped D-Tuning)

D-G-B-G-B-E: Tomatito - »Armonias del Romañe« (Colombianas)

D-G-D-G-B-E: Esteban de Sanlúcar - »Mantilla de Feria« (Fantasía)

$E b-A b-D b-G-B-E$ : Gerardo Núñez: »Templo Del Lucero« (Soleá por Bulerías)

E-A-C\#-A-C\#-F\#: Tomatito - »Callejon de las Canteras« (Tarantas)

$E-A-D-G-A-D: \quad$ Rafael Riqueni - »Agüita clara« (Bulerías)

E-A-D-G\#-C-E: Carlos Piñana - »La voz del viento « (Farruca)

$E-A-D-G-B b-D: \quad$ Paco de Lucía - »Piñonate« (Bulerías)

A-A-D-G-A-D: Paco de Lucía - »La Cañada« (Tangos)

B-A-D-G-B-D\#: Gerardo Núñez - »Remache « (Siguiriya)

$B-A-D-G-B-E: \quad$ Chicuelo - »Cambalache« (Bulerías)

Die Stimmungen erfordern meist komplett neue Fingersätze und -positionen der linken Hand. Eine pragmatische Herangehensweise besteht aber darin, etwa bewährte Griffmuster z.B. für Dominantsept- oder Major 7-Akkorde einfach beizubehalten und so innerhalb der Scordaturas neue spannungsreiche und teils impressionistisch anmutende Färbungen zu erzielen. Hier wird in Flamencomanier kein übergeordnetes zielgerichtetes Harmoniekonzept verfolgt; die Finger suchen sich vielmehr ihren Weg und etablieren so ein eigenes Konzept inklusive Theorie, die eben nicht in verschriftlichter Form, sondern als stilles Wissen vorliegt.

Die alleinige Anwendung von neuen Stimmungen oder alternativen Modi mag selbst noch nicht virtuos sein, sondern vielleicht allenfalls innovativ. Derartiges kann sich auch als Geistesblitz eines unerfahrenen Spielers ereignen. Ein Kriterium kann hier die Häufigkeit sein, die sicherlich in absoluter Dimension nicht zu quantifizieren ist. Der Virtuose aber besitzt die Fähigkeit, einer Idee, einem Impuls aus dem Bereich des Nicht-Greifbaren und Grenzenlosen, seines Wissensschatzes, seiner Erfahrung und seines Verstehens immer wieder zu folgen und Ausdruck zu verleihen und das mit dem 
Anspruch von Konstanz, Häufigkeit und Wiederholung innerhalb eines längeren Zeitraumes. Es ist kein Zufall, dass die technisch stärksten Spieler dieses Potential zur Erneuerung des Genres besitzen und auch ausspielen, wie sich leicht am Beispiel von Gerardo Núñez und Vicente Amigo zeigen lässt. In einem technisch eher unspektakulären Stück wie »Compás Interior « schwingt jene Facette der Virtuosität deshalb unweigerlich mit. Die erwähnten Musiker sind technisch so beschlagen, dass sie ab einem bestimmten Punkt ihrer Karriere ihr virtuoses Potential vornehmlich im Bereich musikalischer Grenzüberschreitungen, Visionsfähigkeit und eigenständiger Kreationen entfalteten. Dabei spielen sie in technischer Hinsicht längst nicht alles aus, was möglich wäre. Etwas flapsig ließe sich sagen: Der Dilettant will und kann nicht, während der Virtuose kann und nicht will. Technisch-handwerkliche Virtuosität bildet letztlich die Grundlage für eine tiefergehende kompositorisch-interpretierende Originalität, welche in meinem Verständnis eine wesentliche Facette von Virtuosität darstellt. Nicht selten wiederum sind solche Momente der Überschreitung eines vorgegebenen Rahmens eng mit den von Moore beschriebenen frictions gekoppelt.

\section{Virtuosität als Erbe des Meisters: Paco de Lucía}

Es ist kaum möglich, über das virtuose Gitarrenspiel im Flamenco zu schreiben, ohne die populärste Figur der letzten fünf Dekaden, Paco de Lucía (1947-2014), unerwähnt zu lassen.

Der Musiker ist das beste Beispiel dafür, wie auf der Grundlage einer spektakulären Technik Virtuosität in dem hier verstandenen erweiterten Sinne entsteht. Mit ihm etablierte sich erstens ein bislang neuartiges technisches und expressives Niveau und zweitens die Integration musikkulturfremder Elemente in den Flamenco. Gerade der zuletzt genannte Faktor löste ab Mitte der 1970er Jahre die Welle des Nuevo Flamenco aus, in der parallel zu den gesellschaftspolitischen Umbrüchen in Spanien eine Vielzahl von genreübergreifenden und grenzüberschreitenden Projekten dem Flamenco einen kosmopolitischen Anstrich gaben. De Lucía redefinierte die Ästhetik des Flamenco und trug darüber hinaus enorm zur Popularisierung der Musik bei. Seine Virtuosität bestand vor allem im kreativen Umgang mit der Tradition und hatte eine substantielle Veränderung im Kern der Musik zur Folge, bei der auf der Basis des Compás rhythmische, harmonische und melodische Prozesse in neuer Weise exponiert und miteinander ins Verhältnis gesetzt wurden. An dieser Stelle sollen nur einige Neuerungen ins Gedächtnis gerufen werden: 
- Mit Paco de Lucía öffnete sich der Flamenco für andere Genres wie Jazz und lateinamerikanische Musik.

- Er führte die ursprünglich aus Peru stammenden Transportkiste und dann als Rhythmusinstrument zweckentfremdeten Cajón in den Flamenco ein. Flöte und E-Bass etablierte er als Standard im Flamenco. Er verwendete Orchesterarrangements und integrierte neue Instrumente wie Bouzouki, Mandoline, Mundharmonika und Trompete.

- Die Basismodelle der andalusischen Kadenz wurden von ihm durch Variantenbildung, Akkordsubstitution, Modulation etc. bereichert und erweitert.

- Kooperation mit Jazz-Musikern, dadurch Aneignung der Grundlagen der Improvisation mit Skalenmaterial aus der Jazzmusik: Ganzton-, HalbtonGanzton und Melodisch-Moll-Skalen. Skalenbasierte Improvisation etablierte sich in der Folge im Flamenco.

- Kollektive Virtuosität: schnelle und komplexe Unisonolinien von E-Bass, Gitarre und Flöte.

Die Ausführungen sollen nun nicht den Eindruck erwecken, es hätte zu Beginn der 1970er Jahre keine weiteren erfindungsreichen Musiker im Flamenco gegeben. Im gleichen Atemzug sollten auch »Serranito und Manolo Sanlúcar erwähnt werden. Paco de Lucía war jedoch die dominierende Figur »because he has opened many doors (Enrique Morente, zit. n. Gamboa 1992: 34). Die Erneuerungen setzten sich in der Folge als anerkannte und quasi-verbindliche Spielnormen durch und wurden als Teil eines weiter aufgefächerten und flexibler werdenden Flamencokanons akzeptiert. Paco de Lucías Wirken beeinflusste die jüngere Generation von Gitarristen so eklatant, dass es sinnvoll scheint, hier von einem Paradigmenwechsel zu sprechen (Krüger 2002: 28). Eine Reihe von Gitarristen übernahmen die Elemente seiner Modernisierung und entwickelten sie kontinuierlich weiter. Neben den bereits erwähnten lange im Musikgeschäft tätigen Spielern wie Pepe Habichuela (*1944) Tomatito (*1958), der Folgegeneration mit Gerardo Nuñez (*1961), Rafael Riqueni (*1962), Juan Manuel Cañizares (*1966), Juan Gómez »Chicuelo« ( ${ }^{* 1968)}$, Vicente Amigo (*1968) sind die heutigen Stars des Toque u.a. Antonio Rey (*1981), Diego del Morao (*1979) und Daní de Morón $\left({ }^{* 1981)}\right.$. Alle hier aufgeführten Musiker sind ausnahmslos brillante Techniker und Virtuosen, die ihr Handwerk bereits in jungen Jahren von Grund auf als Tocaor in der Gesangs- und Tanzbegleitung erlernt haben. Sie sind heute auch solistisch tätig und bespielen trotz einer ökonomisch oft heiklen Situation des Kultur-, Konzert- und Festivalwesens in und außerhalb Spaniens zum Teil mittlere bis große Konzertsäle. Das Erbe de Lucías zeigt 
sich zunächst am offensichtlichsten in der Besetzung und Instrumentierung der musikalischen Formationen sowie der von ihm Mitte der 1980er Jahre eingeführten und bewährten Abfolge und Dramaturgie eines Konzertes. Zunächst spielt der Solist ein bis drei Stücke ohne jegliche Begleitung, anschließend wird die Besetzung sukzessiv vom Duo, Trio bis hin zur vollen Sextett- oder Septettformation in der zweiten Konzerthälfte aufgebaut. Erst hier erreicht das Konzert den musikalisch-dramaturgischen Höhepunkt inklusive Perkussion, Palmas, Tanz, Gesang, Gitarren und Improvisationen. Die Besetzung wird generell flexibel je nach Spielsituation und Finanzierung beliebig reduziert oder erweitert.

\section{Schlussbemerkung}

Die Ausführungen eignen sich sicherlich nicht primär dafür, den Begriff der Virtuosität zu schärfen, ihn einzugrenzen oder aus dem Bereich des Diffusen zu befreien. Sie sind vielmehr ein Plädoyer dafür, inn in seiner Vielgestaltigkeit zu akzeptieren und auf Varianten hinzuweisen, die sich dem Zuschauer oder Zuhörer nicht zwingend offenbaren. Um Höchstleistungen handelt es sich dennoch, welche aber spielerisch-kreativ entwickelt werden und nicht ausschließlich mit den handwerklich orientierten Kategorien und Tugenden des Schwitzens, Übens, Leidens, Verzichtens und Quälens einhergehen. Diese technischen Qualitäten sind allerdings die notwendigen Bedingungen dafür, dass sich die hier geschilderten latenten Facetten mit allen Komponenten der Expressivität, Kommunikation oder des Innovationspotentials zeigen können. Technische Virtuosität ist nur die Grundlage, auf der sich latente Virtuosität entfalten kann.

Die erwähnten Musiker können in der Regel nahezu alles in ihrem Genre spielen und besitzen instrumentale Fähigkeiten, die schwerlich zu überbieten sind. Wenn sich nun einer dieser Gitarristen entschließt, den einen Akkord in einer bestimmten Spielsituation über einen halben Compás einfach zu halten, kann das für diesen Kontext in diesem Moment sehr wohl hochgradig virtuos im Sinne eines kreativen Umgangs mit den Hörererwartungen sein, denn dahinter steht die intuitiv-künstlerische Entscheidung, dies in diesem Moment genauso zu tun und nicht anders. Sicherlich braucht es dafür auch den Hörer und Zuschauer, der dies als virtuos empfindet oder erkennt oder den Musikforscher oder Liebhaber, der darauf hinweist. Einem Anfänger, der den gleichen Akkord zwei Takte hält, weil er keine Alternative kennt, wird man nie Virtuosität attestieren wollen. Natürlich völlig zu Recht, denn die Entscheidungsgrundlage des Spielenden ist eine gänzlich 
andere. Die Qualität des stillen Wissens, das der (intuitiven) Entscheidung vorausgeht, eben genau diesen Akkord zu halten, zeugt von einem anderen Bewusstsein und Fundus an Optionen als bei einem Schüler, dessen Auswahl sich auf zwei Akkorde beschränkt und der sich keiner anderen Möglichkeiten bedienen kann. Die Umsetzung einer außergewöhnlichen oder gar brillanten Idee mit besonderen handwerklichen Mitteln kann also ein Aspekt latenter Virtuosität sein.

Die musikalischen Beispiele waren Beleg dafür, dass sich Virtuosität im zeitgenössischen Flamenco darüberhinaus ganz entscheidend im rhythmischen Bereich manifestiert. Die Grundlage hierfür ist die beschriebene Internalisierung des Compás. Das moderne Gitarrenspiel zeichnet sich darauf aufbauend vor allem durch eine Priorität der rhythmischen Ereignisse wie Síncopas, Contratiempos und Polirítmicos aus. Das äußert sich neben einer perfekt artikulierten Spielweise des Contratiempo beispielsweise auch darin, dass rhythmische Akzente einer Siguiriyas über einer Bulerías gespielt oder der Compás eines Tanguillos mit einer Alegrías verknüpft werden. Dies wäre gar eine Ausweitung des skizzierten Metaflamenco auf den rhythmischen Bereich. Die skizzierten heutigen Entwicklungen im Toque sind im hohen Maße dem Innovationsgeist Paco de Lucía zu verdanken. Er gilt als Personifikation eines Virtuosen schlechthin. Eine seiner bemerkenswerten virtuosen Fähigkeiten lag vor allem darin, auf seine Umwelt zu reagieren, die aktuellen Strömungen und Tendenzen, auch die seiner Musikerkollegen, aufzunehmen und sogleich künstlerisch zu verarbeiten.

Wie lassen sich abschließend die künftigen Entwicklungen des zeitgenössischen Toque in der Post-Paco-Ära im Hinblick auf den Aspekt der Virtuosität skizzieren? Bezüglich des Repertoires dominieren die populären Stile eines überschaubaren Kernkanons, allen voran Bulerías, Tangos und Rumbas. Beliebt bei Gitarristen sind ebenso diejenigen Palos, die sich zur Demonstration einer musterhaften gitarristischen Technik eignen, wie Zapateados oder metrisch freie Stile, die eine Kombination von üppiger präludierender Virtuosität und hoher Expressivität vertragen können wie Granaínas, Tarantas oder Rondeñas. Auf die schweren sublimen, bisweilen tragischen JondoFormen wird zumeist verzichtet, um ein möglichst breites Publikum anzusprechen.

Trotz der offensichtlich wachsenden musikalischen Vielfalt und technischen Perfektion der jüngeren Gitarristen, werden gewisse Tendenzen von manchen kritisch betrachtet. Die aktuelle Ausrichtung der Spielweise der Flamencogitarre birgt die Gefahr, dass erstens das Gitarrenspiel zur reinen Demonstration technischer Meisterschaft und rhythmischer Dominanz mutiert und zweitens, die Grundlagen des Spiels, die Gesangs- und Tanzbeglei- 
tung sowie die Kenntnisse der alten Meister in den Hintergrund geraten. Norberto Torres Cortés äußert sich kritisch im Hinblick auf die Zukunft des Flamencospiels und entdeckt hinsichtlich der verwendeten Rhythmen, Harmonien, Kadenzen, Modulationen, Stimmungen und genreüberschneidenden Fusionen Tendenzen eines Stillstandes, weil alle Parameter bereits von den vielen auf dem Musikmarkt konkurrierenden Gitarristen überbordend ausgereizt wurden (Torres Cortés 2006: 32f.). Dem ist meiner Ansicht nur bedingt zuzustimmen. Das zeitgenössische Gitarrenspiel ist weit davon entfernt, in Erstarrung zu verfallen und nur noch bekannte Formeln zu reproduzieren. Für die Musiker mit dem Anspruch, etwas Neues zu erfinden, ist es zwar zweifelsohne schwieriger als noch zu Beginn der 1970er Jahre. Dennoch darf ihre Kreativität und Originalität nicht unterschätzt werden, da sie immerfort zu neuen stilistischen und ästhetischen Synthesen führt. Einigen Gitarristen wird genau die Verknüpfung aus gefühlsbetontem Spiel und perfekter Technik, d.h. die Brücke zwischen Ausdruck und Artistik vorzüglich gelingen. In kurzen Intervallen erscheint doch immer wieder eine neue Veröffentlichung, die einen Teil der Fachwelt und/oder den Verfasser staunen lässt angesichts der gekonnten Präsentation von Virtuosität als höchste Musikalität und geschmackvolle Innovation. Der Einfallsreichtum des heutigen Toque ist subtiler geworden und die Feinheiten des Spiels zeigen sich, und das ist das zentrale Ergebnis meiner Studie, in latenter Form erst auf den zweiten Blick. Mit Sicherheit wird die weitere Entwicklung des Toque, wie bisher in seiner über 150jährigen Geschichte, ein dezenter und langsamer Prozess werden. Paco de Lucía brachte es kurz vor seinem Tod auf den Punkt: »La evolución está en matices« [»Die Evolution [der Flamencogitarre] vollzieht sich in Nuancen«] (zit. n. Fernández 2010).

\section{Literatur}

Caballero, Ángel Álvarez (1995). La discoteca ideal de flamenco. Barcelona: Planeta.

Faucher, Alain (1994). Arte Clásico Flamenco. Ramón Montoya. Paris: Affedis.

Fernández, David (2010). »Entrevista con Paco de Lucía. >Hacer algo distinto ahora es difícil, falta personalidad.." In: Diario de Sevilla vom 15. August, http://www.diariodesevilla.es/article/entrevistas/767930/hacer/algo/distinto/ es/dificil/ahora/falta/personalidad.html (Stand vom 31.1.2017).

Gamboa, Jose Manuel (1992). »La Guitarra Flamenca. The Flamenco Guitar.«In: La Caña. Nr. 3 (März-Juni), S. 18-36.

Iven, Miguel (1990). "Zwischen zwei Ström(ung)en. Tanzbegleitung zwischen Elbe und Guadalquivir."In: Gitarre spezial. Eine Retrospektive über 10 Jahre Gitarre aktuell. Hg. v. Peter Maier. Hamburg: Verlag Gitarre aktuell, S. 140. 
Kopiez, Reinhard (2004). »Virtuosität als Ergebnis psychomotorischer Optimierung.« In: Musikalische Virtuosität. Hg. v. Heinz von Loesch, Ulrich Mahlert und Peter Rummenhöller. Mainz u.a.: Schott, S. 205-231.

Krüger, Stefan (2002). Die Musikkultur Flamenco. Darstellung, Analyse und Diskurs. Hamburg: Universität Hamburg [Dissertation], http://ediss.sub.uni-hamburg.de/ volltexte/2001/901/pdf/dissertation.pdf (Stand vom 15.1.2017).

Landborn, Adair (2015). Flamenco and Bullfighting: Movement, Passion and Risk in Two Spanish Traditions. Jefferson, NC: McFarland.

Loesch, Heinz von (2004). »Virtuosität als Gegenstand der Musikwissenschaft.« In: Musikalische Virtuosität. Hg. v. Heinz von Loesch, Ulrich Mahlert und Peter Rummenhöller. Mainz u.a.: Schott, S. 11-16.

Manuel, Peter (2006). »Flamenco in Focus. An Analysis of a Performance of Soleares. "In: Analytical Studies in World Music. Hg. v. Michael Tenzer. Oxford: University Press, S. 92-119.

Moore, Allan F. (2012). Song Means: Analysing and Interpreting Recorded Popular Song. Farnham, Surrey: Ashgate.

Schulze, Bernhard-Friedrich (1985). "Ästhetik, Geschichte und Bauweise der Flamencogitarre.« In: Flamenco. Gitano andaluz. Hg. v. Claus Schreiner. Frankfurt/M.: Fischer, S. 142-161.

Small, Christofer (1998). Musicking. The Meanings of Performing and Listening. Middletown, CT: Wesleyan University Press.

Torres Cortés, Norberto (2005). Historia de la guitarra flamenca. El surco, el ritmo y el Compás. Córdoba: Almazura.

Torres Cortés, Norberto (2006). Guitarra Flamenca. Vol. 2. Lo Contemporáneo y otros escritos. Sevilla: Signatura Ediciones de Andalusía.

\section{Diskografie}

Amigo, Vicente (1991). »Maestro Sanlúcar«, »Morente«, »Reino de Silia«. Auf: De mi Corazón al Aire. CBS/Sony, COL 4689321.

Amigo, Vicente (1995). »El Mandaito«. Auf: Vivencias Imaginadas. CBS/Sony, COL 4811972.

Amigo, Vicente (2005). »Campo de la Verdad«. Auf: Un Momento en el Sonido. Sony BMG Music Entertainment, 82876698742.

Amigo, Vicente (2013). »Río de la Seda«. Auf: Tierra. Sony Music, 88765435322.

Chicuelo (2000). »Cambalache«. Auf: Cómplices. Harmonia Mundi, HME 987024.

Isla, Camarón de la / Lucía, Paco de (1972). »Canastera«. Auf: Canastera. Phillips, 6328076.

Morao, Diego del (2010). »Gañanía«. Auf: Orate. Warner Music Spain, 5249835702.

Morón, Dani de (2012). »Immigración«. Auf: Cambio De Sentido. La Voz Del Flamenco, LVF1099.

Nuñez, Gerardo (1987). »Los Caños de la Meca«. Auf: El Gallo azul. Flamenco Accidentales, FA-001.

Nuñez, Gerardo (1997). »Remache«. Auf: Jucal. Alula Records, ALU 1002.

Núñez, Gerardo (2004). "La Habana a Oscuras«, "Siempre es Tarde«, "Soleá de la Luna Coja«, »Templo del Lucero«. Auf: Andando el Tiempo. ACT, ACT 9426-2.

Núñez, Gerardo (2012). »Compás Interior«. Auf: Travesía. ACT, ACT 9534-2.

Lucía, Paco de (1981). »Piñonat«. Auf: Sólo quiero caminar. Philips, 6301030. 
Lucía, Paco de (1987). »La Cañada«. Auf: Sirocco. Mercury, 830913-4.

Piñana, Charlos (1999). »La Voz del Viento«. Auf: Calibiri. Big Bang, BB430CD.

Riqueni, Rafael (1995). »Agüita clara«. Auf: Mi Tiempo. Nuevo, 12658.

Sanlúcar, Esteban de (1954). »Mantilla de Feria«. Auf: Flamenco! RCA Victor, LPM3209.

Sanlúcar, Manolo (1998). »Tercio de Vara«. Auf: Tauromagia. Polygram, 835552.

Tomatito (1991). »Armonias del Romañe«, »Callejon de las Canteras«, »Caminillo Viejo«. Auf: Barrio negro. Nuevos Medios, 13588 LE.

Tomatito (1997). »Barrio Santiago«. Auf: Rosas del Amor. Hispavox, 724385944024.

\begin{abstract}
In contemporary Flamenco guitar-playing virtuosity cannot be reduced to technical perfection alone. Starting off from this premise the article analyses the levels that are included in the concept of virtuosity within the genre under musical aspects. The focus is on selected creative musical artistic tools, generally harder to be identified by the inexperienced recipient or fan and mostly beyond the familiar principle $»$ faster - higher - louder .
\end{abstract}

